\title{
Intrazerebrale Massenblutung: Durafistel aus atypisch entspringender $A$. meningea media
}

\begin{abstract}
Anomalien der hirnversorgenden Gefäße basieren auf Variationen in der embryonalen Entwicklung (Lasjaunias P, Berenstein A. Surgical Neuroangiography, Springer 1987; Bd.1). Eine ganze Reihe von anatomischen Varianten sowohl der supraaortalen als auch der intrakraniellen Gefäße sind bekannt (Schumacher M et al, AJNR 1994; 15: 550). Sie können entscheidend sein für die Planung und Risikoeinschätzung einer neurointerventionellen/neurochirurgischen Therapie, wie am vorliegenden Fallbeispiel gezeigt wird.
\end{abstract}

\section{Fallbericht}

Eine 44jährige Patientin erlitt zwei Wochen vor der stationären Aufnahme einen fokal eingeleiteten generalisierten tonisch-klonischen zerebralen Krampfanfall. Die daraufhin durchgeführte CCT war normal. Die weitere internistische Abklärung ergab bis auf erhöhte Blutdruckwerte keine Auffälligkeiten. Bei mehrfach kontrollierten deutlich erhöhten systolischen und insbesondere diastolischen Blutdruckwerten über $110 \mathrm{~mm} \mathrm{Hg}$ bestand der V.a. eine hypertensive Enzephalopathie als Ursache des zerebralen Krampfanfalls. Es erfolgte daraufhin die medikamentöse Einstellung des arteriellen Hypertonus. Nach Entlassung kam die Patientin wegen eines erneuten zerebralen Krampfanfalls zur Wiederaufnahme. Pektanginöse Beschwerden waren unter Gabe von Nitropräparaten reversibel. Im EKG zeigten sich T-Negativierungen in den Brustwandableitungen V4 bis V6, laborchemisch fanden sich jedoch keine Hinweise auf eine myokardiale Ischämie. Im weiteren Verlauf kam es erneut zu in Folge auftretenden generalisierten zerebralen Krampfanfällen, schließlich zu einer progredienten Vigilanzminderung und schlaffen Hemiparese links sowie zu Strecksynergismen bei mittelweiten, isokoren und prompt reagiblen Pupillen. Im weiteren Verlauf wurde die Patientin komatös. Im CCT wurde eine rechts frontale intrazerebrale Massenblutung nachgewiesen (Größe $6 \times 5 \times 4 \mathrm{~cm}$, s. Abb.1). Die i.a. DSA zeigte eine rechtsseitige durale AV-Fistel hochfrontal, die überwiegend aus einer aus der A.ophthalmica entspringenden A.meningea

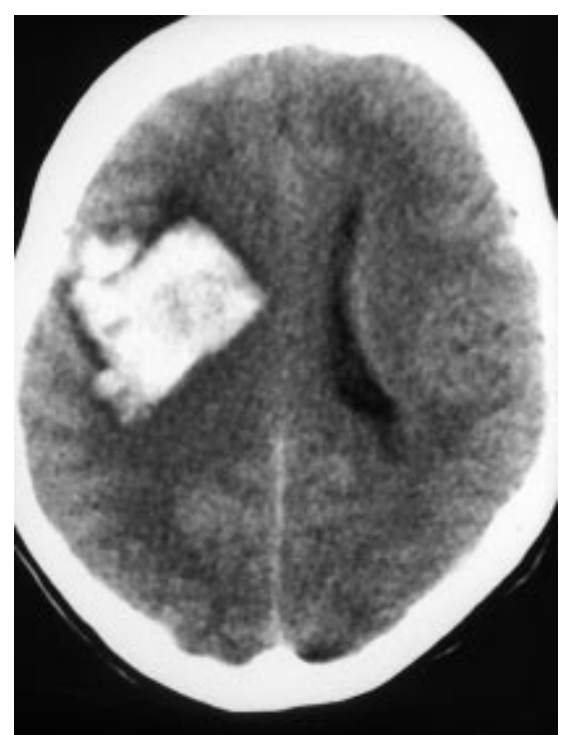

Abb.1 Intrazerebrale rechts frontale Massenblutung mit Oberflächenkontakt.

media rechts versorgt wurde (s. Abb. 2) und einen kleinen Zufluß aus einem Ast der vorderen Falxarterie links aufwies. Die Drainage erfolgte in eine kortikale Vene in unmittelbarer Nachbarschaft zum Sinus sagittalis superior. Angiografisch gab es keinen Hinweis auf eine abgelaufene Thrombose des Sinus sagittalis superior. Die notfallmäßige Ausräumung der Blutung mit operativer Entlastung und die Unterbindung der A.meningea media sowie der übrigen duralen Zuflüsse ergab einen kompletten Verschluß der Fistel (s. Abb. 3). Postoperativ kam es bei einem durch pulmonale Komplikationen erschwerten Verlauf neurologisch zu einer Besserung der Vigilanz und einer Regredienz der linksseitigen Hemiparese.

\section{Diskussion}

Der Anteil duraler Fisteln an den intrakraniellen Gefäßmalformationen beträgt 10-15\%. Die Hauptlokalisation liegt an der Schädelbasis (Sinus transversus, Sinus sigmoideus) sowie am Sinus cavernosus (DeMarco et al., Radiol 1990; 175: 193 - 199), während andere, supratentorielle durale Fisteln (entlang des Sinus sagittalis superior oder des Sinus rectus) eher selten sind. Die duralen Fisteln treten häufig solitär, in etwa 7\% multipel
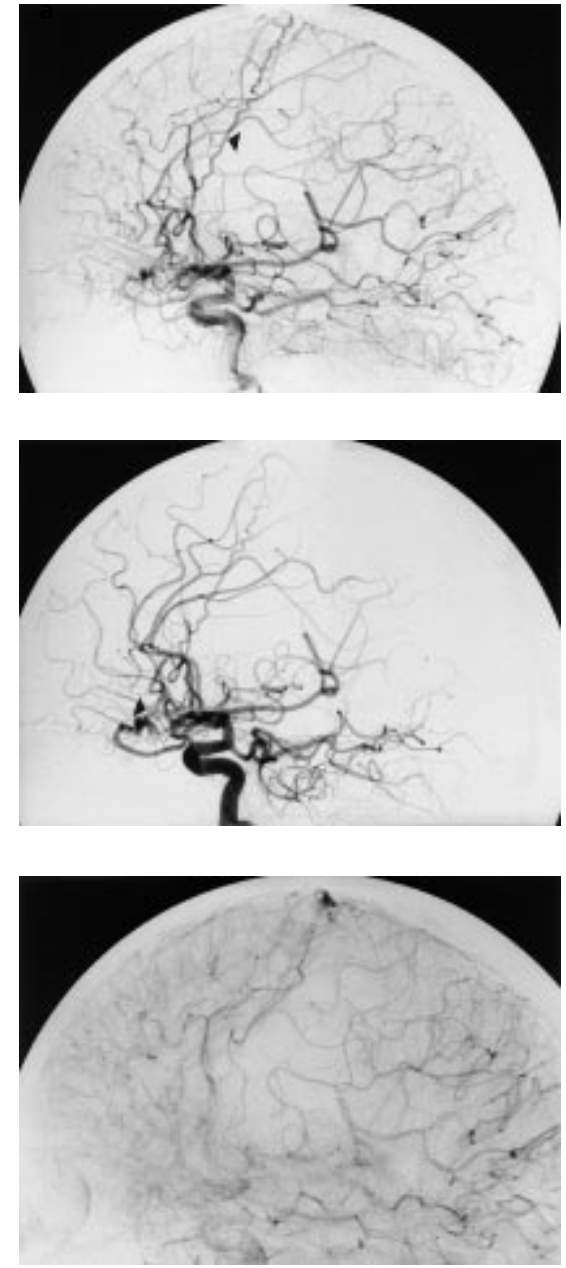

Abb. 2 Angiogramm der A. carotis interna rechts mit fistelversorgender $A$. meningea media, die aus der A. ophthalmica entspringt (s. Pfeile). Normales Angiogramm der A. carotis externa (nicht abgebildet).

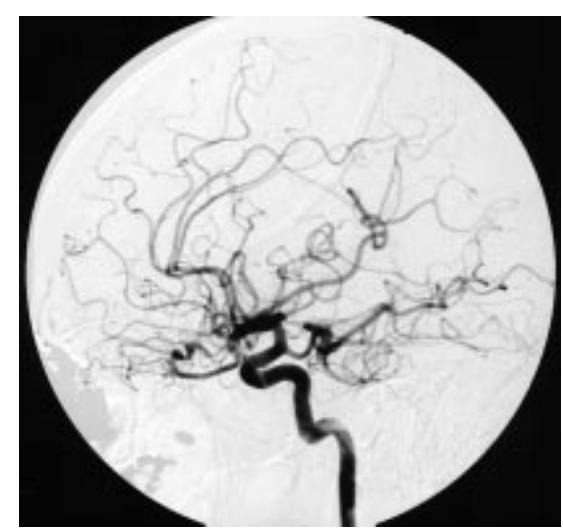

Abb. 3 Postoperatives Angiogramm der A. carotis interna rechts. Ausschaltung der Fistel. 
auf, und bilaterale Zuflüsse sind insbesondere bei duralen Sinus cavernosus Fisteln häufig. Das durchschnittliche Erkrankungsalter liegt bei 55 Jahren. Wenn die durale Fistel supratentoriell lokalisiert ist, hängt die fokale neurologische Symptomatik von der Lokalisation der kortikalen venösen Dysfunktion ab (venöse Hypertension, Thrombose oder Ruptur), und eine kortikale Drainage erhöht das Blutungsrisiko einer duralen Fistel (Awad et al., Neurosurg 1990; 72: 839-850). Die Behandlungsindikation hängt damit u.a. vom venösen Drainagetyp ab. Etwa 25 - 37\% der duralen Fisteln mit kortikaler Drainage werden durch eine intrazerebrale oder subarachnoidale Blutung symptomatisch (Kupersmith MJ, Neurovascular Neuroophthalmology, Springer 1993). Bei jeder intrakraniellen Blutung, insbesondere bei einer Subarachnoidalblutung (SAB) ohne Aneurysmanachweis, muß die gezielte Suche nach einer duralen Fistel als Blutungsquelle erfolgen.

Epileptische Anfälle oder migräneartige Symptome können erste klinische Anzeichen sein. Auch bei unserer Patientin ging zwei Wochen vor Auftreten der intrazerebralen Blutung ein fokal eingeleiteter generalisierter tonisch-klonischer zerebraler Krampfanfall voraus, der Anlaß zu einer CCT-Untersuchung gab, diese jedoch unauffällig war.

Normalerweise entspringt die A. meningea media aus der A. maxillaris und zieht durch das Foramen spinosum in die mittlere Schädelgrube, wo sie sich in den vorderen $R$. frontalis und den hinteren R. parietalis teilt. Die Teilung kann bereits im Foramen spinosum, dicht oberhalb desselben oder weiter distal in der Schädelhöhle erfolgen. Eine ganze Reihe von anatomischen Abgangsvarianten der A. meningea media sind beschrieben worden (Aschen GF Jr et al. AJNR 1980: 1: 45). Die vorliegende Abgangsvariante aus der A.ophthalmica wird mit einer Häufigkeit von $0.5 \%$ aller Fälle angegeben und ist damit die häufigste Abgangsvariante der A. meningea media. Ein unilateraler Visusverlust kann von einem sog. „steal“-Phänomen verursacht sein, wenn eine Gefäßmalformation aus der A.ophthalmica versorgt wird.

Die bei Gefäßanomalien, insbesondere Agenesien gehäuft auftretenden Aneurysmen sind mit Abgangsvarianten der A. meningea media nicht assoziiert.
Obwohl die zum großen Teil aus A. carotis-externa-Zuflüssen versorgten Durafisteln eine Domäne der interventionellen neuroradiologischen Behandlung sind, bestand bei unserer Patientin eine zwingende OP-Indikation, um die raumfordernde Blutung zu entlasten. Unmittelbar nach der Trepanation wurde die A. meningea media unterbunden (s. Abbildungen) und ein weiterer duraler Zufluß aus der vorderen Falxarterie der Gegenseite am Fistelpunkt operativ ausgeschaltet.

Abgesehen von der absoluten OP-Indikation in diesem Fall ist die Indikation zu einer Embolisationsbehandlung streng zu stellen, wenn das Risiko für eine Fehlembolisation der A. ophthalmica aufgrund anatomischer Varianten hoch ist. Wie bei der vorliegenden Durafistelversorgung oder bei einer duralen Tu- morversorgung aus atypisch aus der A. ophthalmica abgehenden Meningealarterien (Götz GF et al. Klin Neurorad 1995; 5: 24) ist daher häufig ein operalives Vorgehen sicherer.

Zusammenfassend können sich Durafisteln sowohl durch eine SAB, ein Subduralhämatom, eine intrazerebrale Blutung oder eine Kombination aus diesen Blutungstypen manifestieren. In seltenen Fällen kann eine Durafistel durch atypisch abgehende meningeale Gefäße versorgt werden. Abgangsvarianten können entscheidend Einfluß auf die Indikation zu einer endovaskulären neuroradiologischen und/oder operativen Therapie nehmen.

J. Klisch, V. v. Velthoven, J. Speer, Freiburg 